\section{Health-related quality of life (SF-36) in back pain: a population-based study, Campinas, São Paulo State, Brazil}

\author{
Qualidade de vida relacionada à saúde (SF-36) \\ e dor lombar: um estudo populacional em \\ Campinas, São Paulo, Brasil
}
Calidad de vida relacionada con la salud (SF-36) respecto al dolor de espalda: un estudio poblacional, Campinas, São Paulo, Brazil

\begin{abstract}
Our study aimed at assessing back pain impact over health-related quality of life (HRQoL). This is a cross-sectional population-based study that analyzed 1,192 adults. The dependent variables were the SF-36 scales and the main independent variables was back pain characterized by location, number of back pain region, intensity, frequency and limitations. Simple and multiple linear regression models were performed to estimate the crude and adjusted beta-coefficients (gender, age, schooling and co-morbidity conditions). Back pain prevalence were $35.4 \%$. For HRQoL, comparing people with/without back pain, we found weak associations for the physical component $(\beta=-3.6)$. However, strong associations were found for physical component $(\beta=-12.4)$ when there were concomitant pain in cervical, dorsal and lumbar sites and also associations with mental health scales. Daily pain was associated with physical $(\beta=-6.8)$ and mental $(\beta=-2.7)$ components. Important impact on physical componente summary was found for intense/very intense pain $(\beta=-7.9)$ and pain with severe limitation $(\beta=-11.5)$. The impacts over HRQoL were strong when back pain was followed by (1) multiple back sites, (2) with pain in mental componente summary, (3) daily complaints, (4) very intense pain and (5) severe limitations; these results have revealed the importance to measure specific factors related to back pain.
\end{abstract}

Back Pain; Quality of Life; Surveys; Epidemiology
Aparecida Mari Iguti 1

Margareth Guimarães 1

Marilisa Berti Azevedo Barros 1

doi: 10.1590/0102-311X00206019

\author{
Correspondence \\ A. M. Iguti \\ Departamento de Saúde Coletiva, Faculdade de Ciências \\ Médicas, Universidade Estadual de Campinas. \\ Rua Tessalia Vieira de Camargo 126, Campinas, SP \\ 13083-887, Brasil. \\ iguti@fcm.unicamp.br \\ 1 Faculdade de Ciências Médicas, Universidade Estadual de \\ Campinas, Campinas, Brasil.
}




\section{Introduction}

Back pain is a prevalent health issue and causes activity limitation, work absenteeism, and productivity loss, impacting quality of life with substantial costs on the healthcare, social, and economic systems ${ }^{1}$. People with neck and/or back pain currently and over the past five years were more often on sick leave and reported worse financial situation, poorer health-related quality of life, low selfesteem and coping ability, with higher degree of perceived stress, vital exhaustion, and depression compared to people without neck and/or back pain 2. Activity-limiting low back pain (LBP) has a lifetime prevalence of about $39 \%$ and a similar annual prevalence of $38 \%$ worldwide. Most people with LBP have experienced recurrent episodes. The global number of individuals with LBP have increased over the decades due to population ageing 3 . Despite the heavy use of all types of healthcare facilities, the prevalence of back pain symptoms and expenditures have been increasing 4 .

Health-related quality of life (HRQoL) is a multidimensional concept, comprising domains of physical, social and mental well-being, and functional ability. The most used generic instruments is the internationally validated Medical Outcomes Study 36-Item Short-Form Health Survey (SF-36). The SF-36 is increasingly used for monitoring the health of populations as a tool for decision-making on health care policies and medical interventions 5 .

Many of the HRQoL studies have been conducted on patients or workers 6,7, and knowledge about the impact of back pain on general populations is scarce. Some researchers have analyzed specific factors related to back pain such as pain location 8.9 , intensity 10,11 , frequency ${ }^{9}$, severity $8,10,12$, and coexistence of pain sites 10 . Others have evaluated musculoskeletal diseases, including back pain 7,12,13,14,15. However, to our knowledge, few authors have evaluated a set of characteristics of back pain in the same study, which is important for a more comprehensive perspective on the subject.

We aimed at analyzing the effects of back pain according to the neck, upper, and lower/sacral sites; number of back pain locations; pain frequency; pain intensity; and physical limitations in the quality of life and health (SF-36) of the adult population of the city of Campinas, São Paulo State, Brazil.

\section{Methods}

This is a cross-sectional, population-based study performed with data of a home-interview survey in the city of Campinas (ISACamp 2014/2015) with data collected in 2014 and early 2015. This survey was conducted with a probabilistic sample, representative of the population living in the urban area of Campinas. Data were collected from three age groups: adolescents, adults, and older adults, for which independent samples were drawn. Data were collected in two stages. At the first stage, 70 census tracts were drawn, proportionally to the number of residences, with 14 census tracts in each of the five health districts of Campinas. At the second stage, the households were drawn. A sample size of 1,000 individuals was defined for the age group of adolescents (10 to 19 years old) and older adults $(\geq 60$ years old). The defined sample for adults (age 20 to 59 years old) was 1,400 individuals. With this size it would be possible to estimate a prevalence of 0.50 (maximum variability for the frequency of events studied) with a $95 \%$ confidence level, sampling error of 5.0 and design effect of 2 . A probability of the number of individuals living at each household by age domain was estimated for five districts, based on the 2010 Demographic Census. The initial sample size estimated was divided by person/household ratio, determining the number of households to be visited: 2,898 for adolescents, 950 for adults, and 3,326 for older adults. This number already considered the non-response rates. All members from the household for that age group were interviewed 16 .

The data were collected using a precoded questionnaire applied by trained interviewers, using tablet computers. The survey questionnaire contained information on demographic and socioeconomic aspects, chronic diseases, accidents and violence, mental health, use of drugs, healthcare services, health-related quality of life and lifestyle. Data on quality of life were obtained using the questionnaire SF-36, version 2. The SF-36 has been translated and validated into Brazilian Portuguese, the second version was validated by Laguardia et al. 17 . SF-36 individual items and domains were conventionally scored: for each of the 8 domains, the item scores were coded, summed, and transformed into a scale from 0 (worst possible health condition measured by the questionnaire) to 100 (best possible health 
condition) ${ }^{17}$. SF-36 also allows estimating two summary components: physical (PCS) and mental (MCS). The permission to use the WHOQOL-BREF Questionnaire was CT120509/OP003754.

We only analyzed the adult population (from 18 to 59 years old). Dependent variables consisted in the eight SF-36 scales and the two summary components. The main independent variables consisted in: back pain complaints/problems (neck, upper, and low back); limitation due to back pain; number of back pain locations (0 to 3 sites); back pain frequency; and back pain intensity. There were other variables for adjustments, considering previous studies $18,19,20$ and the association with our sample: sex (men and women); age range for the adult population (18-29; 20-39; 40-49; 50-59 years); education level (0-8; 9-11; 12 years or over); comorbidity conditions, number of chronic diseases (none; 1 ; 2 ; and 3 or more conditions).

The statistical analysis consisted of estimating means and standard deviations for each of the SF-36 scale scores for each independent variable. Simple and multiple linear regression models were performed to estimate the crude and adjusted $\beta$ coefficients (for sex, age, education level, comorbidity conditions, and number of chronic diseases).

One model was performed for each scale and each group of comparison, and a p-value of less than 0.05 was considered statistically significant. Analyses were performed using the Stata 15.0 software (https://www.stata.com/), incorporating sample, non-response and post-stratification weightings and considering clusters and stratification used in the sample design. All participants signed an informed consent form before the study.

\section{Results}

From the sampled households, the nonresponse rate was of $16.5 \%$ (5.7\% of refusal and $10.8 \%$ lost for absence of household members after more than three visits). Of the identified adult individuals (20 and over) on the sampled households $(1,426)$, the refusal rate was $26.9 \%$, and $2.2 \%$ lost for other reasons. For adolescents (10-19), 1,142 were identified and there were $9 \%$ of refusal and 1,4\% were lost.

Table 1 shows descriptive data regarding sociodemographic characteristics, the presence of back pain, and its site (neck, upper, and low back), intensity, frequency, and physical limitation related to back pain of the study sample. A random sample of 1,192 people aged from 18 to 59 years were studied, with a mean age of 37.1 years (95\%CI: 36.0; 38.1), and 51.4\% women (95\%CI: 48.1; 54.8). Regarding the education level, $31.8 \%$ studied from $0-8$ years; $37.7 \%$, from $9-11$ years; and $30.5 \%$ studied 12 years or over. For chronic diseases, $40.5 \%$ had none and $17.9 \%$ reported 3 or more morbidities. The prevalence of back pain was $35.4 \%$ (95\%CI: $31.5 ; 39.5)$. In the whole populations the prevalence of neck pain was $8.5 \%$, of LBP was $25.7 \%$ and of upper back pain was $9.6 \%$. In the total population, $15.9 \%$ had back pain without physical limitation; $14.6 \%$ had back pain with mild limitation, and $4.8 \%$ had back pain with severe limitation. Among those reporting pain, 54.9\% had limitations.

The mean SF-36 scores decreased in the following scales: physical functioning, role-physical, bodily pain, general health, vitality, and PCS for those reporting back pain with stronger associations with bodily pain $(\beta=-16.4)$ and vitality $(\beta=-6.4)$ (Table 2$)$.

For each of the three sites (neck, upper back, and low back), when comparing the population with or without back pain and specifically analyzing neck pain, we found associations with all scales except for role-emotional, social functioning, mental health, and MCS; for upper back, only role-physical ( $\beta$ $=-7.8)$ and bodily pain $(\beta=-10.3)$ were associated; and for LBP, all scales were associated, except for role-emotional and social functioning (Table 3 ).

By analyzing the number of pain location, for only one site, we found associations with bodily pain $(\beta=-14.5)$ and vitality $(\beta=-5.9)$; for two sites, role-physical $(\beta=-8.4)$, bodily pain $(\beta=-22.3)$, general health $(\beta=-8.9)$, and PCS were associated; for three sites, we observed a strong association with all scales, except for role-emotional, social function, and PCS $(\beta=-12.4)$. An increased decline of SF-36 scores were observed with the increase of the number of pain location (Table 4).

By comparing "no back pain" with "back pain frequency", we found associations with bodily pain and PCS, and we also verified that the higher the frequencies, the stronger the associations. For daily back pain, associations were found for all scales, except for role-emotional. The beta coefficient for PCS was -6.8 ( $\mathrm{p}<0.001)$ and for MCS, $-2.7(\mathrm{p}=0.041)$. When analyzing intensity, we found asso- 
Table 1

Descriptive characteristics of the population.

\begin{tabular}{|c|c|c|c|}
\hline Variable & $\mathbf{n}$ & $\%$ & $95 \% \mathrm{Cl}$ \\
\hline \multicolumn{4}{|l|}{ Sex } \\
\hline Women & 556 & 51.4 & $48.1 ; 54.8$ \\
\hline Men & 636 & 48.6 & $45.2 ; 51.9$ \\
\hline \multicolumn{4}{|l|}{ Age group (years) } \\
\hline $18-29$ & 454 & 30.8 & $26.9 ; 34.9$ \\
\hline $30-49$ & 274 & 28.0 & $24.6 ; 31.6$ \\
\hline $40-59$ & 464 & 41.3 & $36.8 ; 45.9$ \\
\hline \multicolumn{4}{|l|}{ Education level (years) } \\
\hline $0-8$ & 397 & 31.8 & $26.6 ; 37.5$ \\
\hline 9- 11 & 509 & 37.7 & $33.6 ; 42.0$ \\
\hline 12 or over & 285 & 30.5 & $24.8 ; 36.9$ \\
\hline \multicolumn{4}{|l|}{ Number of chronic diseases } \\
\hline None & 477 & 40.5 & $36.1 ; 45.1$ \\
\hline 1 or 2 & 465 & 41.6 & $37.9 ; 45.4$ \\
\hline 3 or over & 194 & 17.9 & $15.0 ; 21.2$ \\
\hline \multicolumn{4}{|l|}{ Number of non-diagnosed health issue } \\
\hline None & 370 & 31.9 & $28.3 ; 35.7$ \\
\hline 1 & 347 & 29.1 & $26.3 ; 32.0$ \\
\hline 2 & 238 & 20.2 & $17.9 ; 22.7$ \\
\hline 3 or over & 234 & 18.8 & $15.9 ; 22.2$ \\
\hline \multicolumn{4}{|l|}{ Back pain } \\
\hline No & 785 & 64.6 & $60.5 ; 68.5$ \\
\hline Yes & 406 & 35.4 & $31.5 ; 39.5$ \\
\hline \multicolumn{4}{|l|}{ Physical limitation for back pain } \\
\hline None & 173 & 15.9 & $13.2 ; 19.1$ \\
\hline Mild limitation & 169 & 14.6 & $11.9 ; 17.9$ \\
\hline Severe limitation & 64 & 04.8 & $3.6 ; 6.4$ \\
\hline \multicolumn{4}{|l|}{ Back pain location * } \\
\hline Neck & 106 & 8.5 & $7.1 ; 10.3$ \\
\hline Upper back pain & 118 & 9.6 & $8.0 ; 11.5$ \\
\hline Low back pain (including sacral region) & 289 & 25.7 & $22.7 ; 29.1$ \\
\hline \multicolumn{4}{|l|}{ Number of back pain locations } \\
\hline 1 & 324 & 28,8 & $24.9 ; 33.1$ \\
\hline 2 & 57 & 4.7 & $3.3 ; 6.5$ \\
\hline 3 & 25 & 1.9 & $1.2 ; 3.1$ \\
\hline \multicolumn{4}{|l|}{ Frequency of back pain } \\
\hline Sometimes & 176 & 16.6 & $13.5 ; 20.4$ \\
\hline Some days of the week & 129 & 10.7 & $8.9 ; 12.7$ \\
\hline Daily & 99 & 7.9 & $6.4 ; 9.8$ \\
\hline \multicolumn{4}{|l|}{ Intensity of back pain } \\
\hline Weak/Mild & 97 & 9.0 & $7.0 ; 11.4$ \\
\hline Moderate & 165 & 14.4 & $12.5 ; 16.5$ \\
\hline Intense/Very intense & 144 & 12.1 & $9.8 ; 14.7$ \\
\hline
\end{tabular}

95\%Cl: 95\% confidence interval.

* Perceptual among total population; one person can have multiple back pain locations. 
Table 2

Standardized scores and beta coefficients of the Medical Outcomes Study 36-Item Short-Form Health Survey (SF-36)

health-related quality of life scale for population groups with back pain and without back pain.

\begin{tabular}{|c|c|c|c|c|}
\hline \multirow[t]{2}{*}{ SF-36 domains } & \multicolumn{2}{|c|}{ Mean SF-36 scores (SE) } & \multirow[t]{2}{*}{$\beta$ coefficients * } & \multirow[t]{2}{*}{$95 \% \mathrm{Cl}$} \\
\hline & $\begin{array}{c}\text { No back pain } \\
(n=785)\end{array}$ & $\begin{array}{c}\text { Back pain } \\
(n=406)\end{array}$ & & \\
\hline Physical functioning & $94.4(0.60)$ & $87.7(1.26)$ & $-3.4 * *$ & $-6.2 ;-0,7$ \\
\hline Role-physical & $93.0(0.75)$ & $83.6(1.67)$ & $-5.0 * \star$ & $-8.5 ;-1.6$ \\
\hline Bodily pain & $85.6(1.08)$ & $64.3(1.53)$ & $-16.4 * \star$ & $-20.0 ;-12.8$ \\
\hline General health & $83.9(1.03)$ & $76.1(1.09)$ & $-4.5^{* \star}$ & $-7.7 ;-1.3$ \\
\hline Vitality & $80.7(0.71)$ & $69.8(1.20)$ & -6.4 ** & $-9.1 ;-3.8$ \\
\hline Role-emotional & $92.4(0.84)$ & $87.4(1.43)$ & -0.5 & $-4.0 ; 3.1$ \\
\hline Social functioning & $91.0(0.83)$ & $84.7(1.35)$ & -0.6 & $-3.7 ; 2.6$ \\
\hline Mental health & $80.5(0.84)$ & $72.4(1.27)$ & $-3.0 * * *$ & $-5.7 ;-0.3$ \\
\hline PCS & $53.1(0.26)$ & $40.1(0.60)$ & $-3.6 * *$ & $-4.8 ;-2.4$ \\
\hline MCS & $51.0(0.42)$ & $48.2(0.66)$ & -0.3 & $-1.8 ; 1.16$ \\
\hline
\end{tabular}

95\%Cl: 95\% confidence interval; MCS: mental component summary; PCS: physical component summary; SE: standard error.

* $\beta$ coefficients estimated from multiple linear regression, including sex, age group, education level, and number of comorbidities;

$\star * \mathrm{p}<0.05$;

$\star * \star p<0.01$.

ciation for intense/very intense back pain in all scales, except for role-emotional, social function, and MCS, with strong associations with bodily pain $(\beta=-26.4)$, role-physical $(\beta=-15.7)$, physical functioning ( $\beta=-12.6)$, and vitality $(\beta=-12.6)$. Individuals reporting back pain without limitations featured bad scores in bodily pain $(\beta=-11.9)$ and vitality $(\beta=-3.3)$; and those with mild limitations had worse scores in bodily pain, vitality, mental health, and PCS. Strongest associations were found comparing severe limitations with no back pain in all scales, mainly in pain $(\beta=-28.7)$, role-physical $(\beta=-28.3)$, and physical functioning $(\beta=-21.0)$. The decline of the SF-36 scores increased with the increase in pain frequency and intensity and with the degree of reported limitation (Table 5). 
Table 3

Mean differences in Medical Outcomes Study 36-Item Short-Form Health Survey (SF-36) scores between adults with no pain and pain in specific sites.

\begin{tabular}{|c|c|c|c|}
\hline \multirow[t]{2}{*}{ SF-36 domains } & \multirow{2}{*}{$\begin{array}{c}\text { No back pain } \\
\text { Mean (SE) }\end{array}$} & \multicolumn{2}{|c|}{ Pain } \\
\hline & & Adjusted $\beta$ coefficients * & $95 \% \mathrm{Cl}$ \\
\hline \multicolumn{4}{|l|}{ Neck $(n=106)$} \\
\hline Physical functioning & $93.1(0.62)$ & $-5.8 * *$ & $-10.5 ;-1.16$ \\
\hline Role-physical & $91.0(0.90)$ & $-8.8 * * *$ & $-14.5 ;-3.2$ \\
\hline Bodily pain & $80.2(1.02)$ & $-16.6 * * *$ & $-22.1 ;-11.4$ \\
\hline General health & $82.2(0.83)$ & $-7.9 * \star \star$ & $-12.9 ;-2.9$ \\
\hline Vitality & $77.9(0.71)$ & $-6.3 * * *$ & $-10.0 ;-2.6$ \\
\hline Role-emotional & $91.3(0.86)$ & -1.8 & $-6.8 ; 3.3$ \\
\hline Social functioning & $89.6(0.76)$ & -2.2 & $-8.9 ; 4.3$ \\
\hline Mental health & $78.5(0.76)$ & -3.2 & $-7.6 ; 1.2$ \\
\hline PCS & $51.9(0.28)$ & $-4.9 * * *$ & $-7.0 ;-2.9$ \\
\hline MCS & $50.3(0.40)$ & -0.3 & $-2.8 ; 2.2$ \\
\hline \multicolumn{4}{|l|}{ Upper back $(n=118)$} \\
\hline Physical functioning & $92.8(0.67)$ & -3.6 & $-7.3 ; 0.2$ \\
\hline Role-physical & $90.8(0.90)$ & $-7.8 * * *$ & $-12.7 ;-2.8$ \\
\hline Bodily pain & $79.5(1.07)$ & $-10.3 * * *$ & $-15.9 ;-4.6$ \\
\hline General health & $81.7(0.77)$ & -2.6 & $-6.6 ; 1.3$ \\
\hline Vitality & $77.6(0.71)$ & -3.4 & $-8.9 ; 2.0$ \\
\hline Role-emotional & $91.1(0.86)$ & -1.2 & $-7.5 ; 5.0$ \\
\hline Social functioning & $89.4(0.81)$ & -3.0 & $-9.8 ; 3.7$ \\
\hline Mental health & $78.4(0.76)$ & -3.7 & $-8.0 ; 0.7$ \\
\hline PCS & $51.8(0.30)$ & $-2.9 * * *$ & $-4.9 ;-0.8$ \\
\hline MCS & $50.3(0.42)$ & -0.8 & $-4.0 ; 2.4$ \\
\hline \multicolumn{4}{|c|}{ Low back [including sacral region] $(n=289)$} \\
\hline Physical functioning & $93.8(0.67)$ & $-4.2 \#$ & $-7.7 ;-0.8$ \\
\hline Role-physical & $92.1(0.84)$ & $-5.0 \#$ & $-9.2 ;-0.9$ \\
\hline Bodily pain & $83.5(1.04)$ & $-16.7 * * *$ & $-21.1 ;-12.6$ \\
\hline General health & $83.2(0.91)$ & $-5.6 * * *$ & $-8.4 ;-3.0$ \\
\hline Vitality & $79.5(0.76)$ & $-7.1 * * *$ & $-10.5 ;-3.4$ \\
\hline Role-emotional & $91.9(0.85)$ & -1.0 & $-3.9 ; 2.1$ \\
\hline Social functioning & $90.2(0.87)$ & -0.7 & $-4.2 ; 2.6$ \\
\hline Mental health & $79.5(0.77)$ & $-3.2 \#$ & $-5.9 ;-0.3$ \\
\hline PCS & $52.6(0.29)$ & -3.9 & $-5.5 ;-2.3$ \\
\hline MCS & $50.6(0.41)$ & -0.5 & $-1.8 ; 0.9$ \\
\hline
\end{tabular}

95\%Cl: 95\% confidence interval; MCS: mental component summary; PCS: physical component summary; SE: standard error.

* $\beta$ coefficients estimated from multiple linear regression, including sex, age group, education level, and number of comorbidities;

** $\mathrm{p}<0.01$;

$\star * \star p<0.001$;

$\# \mathrm{p}<0.05$. 


\section{Table 4}

The Medical Outcomes Study 36-Item Short-Form Health Survey (SF-36) mean scores of adults with no pain and with one, two, and three pain locations.

\begin{tabular}{|c|c|c|c|c|c|c|c|}
\hline \multirow[t]{4}{*}{ SF-36 domains } & \multirow{4}{*}{$\begin{array}{l}\text { No back pain } \\
\text { (n = 785) } \\
\text { Mean (SE) }\end{array}$} & \multicolumn{6}{|c|}{ With back pain $[\beta$ coefficients * $(95 \% \mathrm{Cl})]$} \\
\hline & & \multirow{2}{*}{\multicolumn{2}{|c|}{$\begin{array}{c}1 \text { site } \\
(n=324)\end{array}$}} & \multirow{2}{*}{\multicolumn{2}{|c|}{$\begin{array}{c}2 \text { sites } \\
(n=57)\end{array}$}} & \multirow{2}{*}{\multicolumn{2}{|c|}{$\begin{array}{l}3 \text { sites } \\
(n=25)\end{array}$}} \\
\hline & & & & & & & \\
\hline & & $\beta$ coefficients * & $95 \% \mathrm{Cl}$ & $\beta$ coefficients * & $95 \% \mathrm{Cl}$ & $\beta$ coefficients * & $95 \% \mathrm{Cl}$ \\
\hline Physical functioning & $94.4(0.60)$ & -2.37 & $-5.5 ; 0.8$ & -5.08 & $-10.8 ; 0.8$ & $-17.7 \star \star$ & $-30.9 ;-4.5$ \\
\hline Role-physical & $93.0(0.75)$ & -3.34 & $-7.1 ; 0.4$ & $-8.43 * \star$ & $-16.1 ;-0,7$ & $-26.3 * \star *$ & $-42,1 ;-10.5$ \\
\hline Bodily pain & $85.5(1.08)$ & $-14.5 \#$ & $-18.0 ;-10.9$ & $-22.3 \#$ & $-29.6 ;-15.0$ & $-34.8 \#$ & $-48.1 ;-21.5$ \\
\hline General health & $83.9(1.03)$ & -3.10 & $-6.7 ; 0.5$ & $-8.9 * * *$ & $-15.3 ;-2.6$ & $-17.1 * \star \star$ & $-26.9 ;-7.3$ \\
\hline Vitality & $80.7(0.71)$ & $-5.9 \#$ & $-9.0 ;-2.8$ & -6.23 & $-12.8 ; 0.3$ & $-17.19 * \star \star$ & $-26.5 ;-7.8$ \\
\hline Role-emotional & $92.4(0.84)$ & 0.08 & $-3.8 ; 4.0$ & -1.75 & $-9.0 ;-5.5$ & -7.08 & $-18.1 ; 4.0$ \\
\hline Social functioning & $90.9(0.83)$ & 0.16 & $-3.3 ; 3.6$ & -2.48 & $-11.2 ; 6.3$ & -8.97 & $-22.3 ; 4.4$ \\
\hline Mental health & $80.5(0.84)$ & -2.46 & $-5.3 ; 0.4$ & -3.19 & $-8.1 ; 1.7$ & $-12.38 * *$ & $-22.4 ;-2.4$ \\
\hline PCS & $53.1(0.26)$ & -1.21 & $-4.2 ;-1.6$ & $-4.26 * \star \star$ & $-7.5 ;-2.7$ & $-12.43 \#$ & $-17.8 ;-5.7$ \\
\hline MCS & $51.0(0.42)$ & 0.55 & $-1.9 ; 1.4$ & -0.55 & $-2.8 ; 2.6$ & -3.87 & $-8.3 ; 2.6$ \\
\hline
\end{tabular}

95\% Cl: 95\% confidemce interval; MCS: mental component summary; PCS: physical component summary; SE: standard error.

* $\beta$ coefficients estimated from multiple linear regression, including sex, age group, education level, and number of comorbidities;

** $\mathrm{p}<0.001$;

$\star * \star p<0.01 ;$

$\# \mathrm{p}<0.05$.

\section{Discussion}

The prevalence of back pain in our study is similar to the average in international studies on general adult population 1,3,21, although there are more findings in low and middle-income countries ${ }^{1}$. For pain locations, the highest frequency was in low back, followed by neck and thoracic regions, as other study 22 .

We observed weakest correlations with back pain and physical functioning and no correlations with role-emotional and social functioning when comparing the presence and absence of back pain. These results were in line with Rezai et al. ${ }^{8}$, who conducted a neck pain study. Bodily pain scores were the poorest as expected, since people experiencing back pain are more likely to have worse scores. Negative relationship of back pain with physical health and PCS is in accordance with the results of other studies 9,11,12,13,23. Authors of a study on a large French population also found association with MCS 23.

We found association with a mental component of SF-36 only for daily pain and a strong association between LBP and mental health scales for three pain sites. Lower scores in PCS were associated with higher pain frequency in all scales. Scores in overall physical and mental components of HRQoL were substantially lower when back pain was frequent, compared with no back pain. Our data are in line with those of a Croatian study 9 .

Dominick et al. 10 found significant lower SF-36 mean scores for those reporting chronic pain regarding all SF-36 domains in comparison with those without chronic pain. They also observed crude differences close to 20 points comparing no pain with pain in at least one region, in the scales of physical functioning and role-physical. In other study, researchers found that musculoskeletal pain is independently associated with PCS, pain intensity, number of locations, hip-knee pain, self-reported rheumatic disorders, LBP, and age; moreover, intensity of musculoskeletal pain was one of the predictors of worse physical health 7 .

According to Ogunlana et al. 24, LBP also affected more physical health components than mental health components, and the HRQoL of LBP patients worsened with increasing pain severity and disability. Authors of a follow-up Canadian study comparing people with neck pain and no neck pain 
Table 5

The Medical Outcomes Study 36-Item Short-Form Health Survey (SF-36) mean scores of adults according to back pain frequency, intensity, and limitations due to back pain.

\begin{tabular}{|c|c|c|c|c|c|c|c|}
\hline \multirow[t]{3}{*}{ SF-36 domains } & \multirow{3}{*}{$\begin{array}{l}\text { No back pain } \\
\begin{array}{c}(n=785) \\
\text { Mean (SE) }\end{array}\end{array}$} & \multicolumn{6}{|c|}{ With back pain } \\
\hline & & \multicolumn{2}{|c|}{$\begin{array}{l}\text { Sometimes } \\
\qquad(n=176)\end{array}$} & \multicolumn{2}{|c|}{$\begin{array}{l}\text { Some days of the week } \\
\qquad(n=129)\end{array}$} & \multicolumn{2}{|c|}{$\begin{array}{c}\text { Daily } \\
(n=199)\end{array}$} \\
\hline & & $\beta$ coefficients * & $95 \% \mathrm{Cl}$ & $\beta$ coefficients * & $95 \% \mathrm{Cl}$ & $\beta$ coefficients * & $95 \% \mathrm{Cl}$ \\
\hline Physical functioning & $94.4(0.60)$ & 0.17 & $-2.5 ; 2.8$ & -5.5 & $-11.9 ; 0.9$ & $-9.9 * *$ & $-16.9 ;-3.7$ \\
\hline Role-physical & $93.0(0.75)$ & -1.3 & $-4 ; 6 ; 2.0$ & -6.7 & $-14.4 ; 1.0$ & $-12.8 * *$ & $-20.6 ;-5.0$ \\
\hline Bodily pain & $85.5(1.08)$ & $-10.0 * * *$ & $-14.5 ;-5.6$ & $-20.5 *$ & $-26.6 ;-14.5$ & $-27.9 * \star \star$ & $-34.7 ;-21.1$ \\
\hline General health & $83.9(1.03)$ & -2.2 & $-6.3 ; 2.0$ & $-6.5 * *$ & $-11.4 ;-1.7$ & $-7.6 * *$ & $-12.0 ;-3.2$ \\
\hline Vitality & $80.7(0.71)$ & -3.6 & $-7.2 ; 0.03$ & $-6.4 * *$ & $-10.1 ;-2.7$ & $-14.6 * * *$ & $-19.6 ;-9.6$ \\
\hline Role-emotional & $92.4(0.84)$ & -0.5 & $-5.2 ; 4.3$ & 1.4 * & $-3.3 ; 6.0$ & -3.3 & $-8.8 ; 2.2$ \\
\hline Social functioning & $90.9(0.83)$ & 0.98 & $-3.5 ; 5.5$ & 3.0 & $-1.0 ; 6.9$ & $-8.9 * *$ & $-15.4 ;-2.3$ \\
\hline Mental health & $80.5(0.84)$ & -1.6 & $-4.7 ; 1.6$ & -1.4 & $-5.4 ; 2.6$ & $-8.8 * *$ & $-14.4 ;-3.2$ \\
\hline PCS & $53.1(0.26)$ & $-1.4 \#$ & $-2.9 ;-1.0$ & $-5.5 * \star \star$ & $-8.3 ; 2.8$ & $-6.8 * * *$ & $-9.6 ;-4.5$ \\
\hline MCS & $51.0(0.42)$ & -0.17 & $-2.5 ; 2.0$ & 1.2 & $-0.4 ; 2.6$ & $-2.7 \#$ & $-5.8 ;-0.4$ \\
\hline \multirow[t]{3}{*}{ SF-36 domains } & No back pain & \multicolumn{6}{|c|}{ With back pain } \\
\hline & $(n=785)$ & \multicolumn{2}{|c|}{$\begin{array}{c}\text { Weak/Mild } \\
(n=97)\end{array}$} & \multicolumn{2}{|c|}{$\begin{array}{l}\text { Moderate } \\
(n=165)\end{array}$} & \multicolumn{2}{|c|}{$\begin{array}{c}\text { Intense/Very intense } \\
\qquad(n=144)\end{array}$} \\
\hline & Mean (SE) & $\beta$ coefficients * & $95 \% \mathrm{Cl}$ & $\beta$ coefficients * & $95 \% \mathrm{Cl}$ & $\beta$ coefficients * & $95 \% \mathrm{Cl}$ \\
\hline Physical functioning & $94.4(0.60)$ & 1.88 & $-0.9 ; 4.6$ & 0.25 & $-2.7 ; 3.2$ & $-12.6 * * *$ & $-18.5 ;-6.8$ \\
\hline Role-physical & $93.0(0.75)$ & $2.7 \#$ & $0.04 ; 5.4$ & -2.0 & $-6.0 ; 2.0$ & $-15.7 * \star *$ & $-23.1 ;-8.4$ \\
\hline Bodily pain & $85.5(1.08)$ & -4.07 & $-9.3 ; 1.2$ & -17.03 * & $-22.0 ;-12.1$ & $-26.4 * * *$ & $-33.4 ;-19.4$ \\
\hline General health & $83.9(1.03)$ & -1.4 & $-5.4 ; 2.6$ & -2.4 & $-6.5 ; 1.7$ & $-9.7 * * *$ & $-13.9 ;-5.5$ \\
\hline Vitality & $80.7(0.71)$ & -3.3 & $-7.6 ; 1.1$ & -3.8 & $-7.7 ; 0.0$ & $-12.6 * * *$ & $-16.4 ;-8.7$ \\
\hline Role-emotional & $92.4(0.84)$ & 1.9 & $-3.8 ; 7.7$ & 1.2 & $-2.8 ; 5.4$ & -4.8 & $-10.4 ; 0.7$ \\
\hline Social functioning & $90.9(0.83)$ & 4.0 & $-2.0 ; 10.1$ & -0.6 & $-4.6 ; 3.2$ & -4.5 & $-10.0 ; 0.9$ \\
\hline Mental health & $80.5(0.84)$ & 0.17 & $-3.3 ; 3.7$ & -1.62 & $-4.9 ; 1.7$ & $-7.6 * *$ & $-11.9 ;-3.2$ \\
\hline PCS & $53.1(0.26)$ & -0.4 & $-1.5 ; 1.2$ & $-2.6 * *$ & $-4.3 ;-1.0$ & $-7.9 * * *$ & $-10.4 ;-5.3$ \\
\hline MCS & $51.0(0.42)$ & 0.28 & $-2.4 ; 3.0$ & 0.1 & $-1.8 ; 2.0$ & -1.5 & $-3.4 ; 0.5$ \\
\hline \multirow[t]{3}{*}{ SF-36 domains } & \multicolumn{3}{|l|}{ No back pain } & \multicolumn{2}{|c|}{ With back pain } & \multirow{2}{*}{\multicolumn{2}{|c|}{$\begin{array}{l}\text { Severe limitation } \\
(n=164)\end{array}$}} \\
\hline & $(n=785)$ & \multicolumn{2}{|c|}{$\begin{array}{l}\text { No limitation } \\
\quad(n=173)\end{array}$} & \multicolumn{2}{|c|}{$\begin{array}{l}\text { Mild limitation } \\
\quad(n=169)\end{array}$} & & \\
\hline & Mean (SE) & $\beta$ coefficients * & $95 \% \mathrm{Cl}$ & $\beta$ coefficients * & $95 \% \mathrm{Cl}$ & $\beta$ coefficients * & $95 \% \mathrm{Cl}$ \\
\hline Physical functioning & $94.4(0.60)$ & 0.51 & $-2.3 ; 3.4$ & -3.21 & $-7.5 ; 1.1$ & $-21.0 * * *$ & $-30.7 ;-11.3$ \\
\hline Role-physical & $93.0(0.75)$ & -0.7 & $-2.9 ; 2.9$ & -4.4 & $-9.8 ; 0.9$ & $-28.3 * * *$ & $-38.7 ;-18.1$ \\
\hline Bodily pain & $85.5(1.08)$ & $-11.9 * * *$ & $-16.3 ;-7.4$ & $-18.3 * * *$ & $-23.1 ;-13.5$ & $-28.7 * * *$ & $-37.6 ;-18.8$ \\
\hline General health & $83.9(1.03)$ & -0.9 & $-4.0 ; 2.2$ & -5.2 & $-10.5 ; 0.1$ & $-16.9 *$ & $-23.6 ;-10.2$ \\
\hline Vitality & $80.7(0.71)$ & $-3.3 \#$ & $-6.6 ;-0.3$ & $-7.8 *$ & $-11.2 ;-4.3$ & $-14.9 * * *$ & $-21.3 ;-8.1$ \\
\hline Role-emotional & $92.4(0.84)$ & 2.8 & $-0.1 ; 5.8$ & -0.4 & $-6.2 ; 5.4$ & $-14.8 * *$ & $-24.4 ;-5.2$ \\
\hline Social functioning & $90.9(0.83)$ & 1.41 & $-2.4 ; 5.1$ & -0.11 & $-4.6 ; 4.4$ & $-10.6 \#$ & $-20.5 ;-0.7$ \\
\hline Mental health & $80.5(0.84)$ & -0.4 & $-4.2 ; 3.0$ & $-4.7 * \star *$ & $-8.6 ;-0.7$ & $-7.8 \#$ & $-13.6 ;-1.6$ \\
\hline PCS & $53.1(0.26)$ & $-1.75 * *$ & $-3.1 ;-0.4$ & $-3.63 * * *$ & $-5.3 ;-2.1$ & $-11.48 * * *$ & $-15.4 ;-8.3$ \\
\hline MCS & $51.0(0.42)$ & 0.066 & $-1.2 ; 2.3$ & -0.8 & $-3.2 ; 1.2$ & -2.65 & $-6.6 ; 0.4$ \\
\hline
\end{tabular}

95\%Cl: 95\% confidemce interval; MCS: mental component summary; PCS: physical component summary; SE: standard error.

* $\beta$ coefficients estimated from multiple linear regression, including sex, age group, education level, and number of comorbidities;

** $\mathrm{p}<0.01$;

$* * * p<0.05$;

$\# p<0.001$. 
showed worse physical HRQoL after six months to people with neck pain, and was negatively associated with physical components, but not mental componentes 25 .

The number of pain sites is associated with worse conditions, not only for HRQoL; authors of a Norwegian follow-up study showed that an increasing number of chronic pain sites were inversely associated with probability of recovery, and the number of chronic pain sites showed a strong doseresponse influence in the prognosis of chronic LBP 26. Moreover, authors of a study on 12 musculoskeletal diseases (including back-related issues) found significantly lower scores in all SF-36 dimensions than those without musculoskeletal diseases, especially for physical functioning, role limitations due to physical problems, and bodily pain 13 .

Authors of a Japanese study showed that people with LBP reported more problems in all dimensions of quality of life with significant differences in both physical and mental domains 6 . They estimated that LBP causes loss of quality-adjusted life years (QALYs) accounting for 1.35 per 1,000 inhabitants, substantially depriving the quality of life of the population 6 . In another study, researchers showed that LBP causes activity limitation and participation restriction 24.

We found that HRQoL scores were similar between the "no pain group" and the "pain group without limitation", probably because pain was less severe (less frequent and less intense). Surprisingly, back pain even with minimal limitation was associated with poorer quality of life in the bodily pain domain, and particularly in vitality and mental health scales. Observing the impact of back pain on three back locations and their outcomes, associations between severity of physical limitation and worse HRQoL are evident, which corroborate a study by Stewart et al. 1, who found higher intensity pain associated with worse disability scores and with comorbid chronic conditions.

Back pain with severe limitation was associated with worse quality of life, mainly in the role-physical, bodily pain, and physical functioning domains with differences among scores (adjusted beta coefficient) above 20.0 points. Moreover, the higher the limitation, the worst the scores in all scales. These findings are in line with a chronic back pain study, whose authors found association with decrease in HRQoL regarding all domains of the SF-36, with physical domains being more affected than the mental ones 12 . An Iranian study showed that LBP causes disability and limitations with impacts on the dimensions of quality of life such as role-physical, vitality, mental health, and general health 27 .

For Ogunlana et al. 24, the increasing incidence of disability and pain intensity in LBP patients was a major predictor of poor HRQoL. Back pain is one of the chronic diseases that most impacted health-related quality of life 5. The 2010 Global Burden of Disease Study ranked LBP as the condition with the highest number of years lived with disability (YLDs) and the sixth in terms of DALYs. In 1990, the global burden of YLDs due to back pain in adults aged 50-69 years was 59\% in developing countries, increasing to $67 \%$ in 2010 . Almost $5 \%$ of adults are significantly disabled by neck pain for a six-month period. Common during the most productive years of a person's life, neck pain impacts people's general health and HRQoL 1 .

In our study, we emphasized strong associations between back pain and vitality, regardless pain frequency and limitations. Vitality involves physical and mental dimensions of health 28 and includes questions concerning being full of energy, feeling worn out and feeling tired. Three points on vitality scores can increase in 38\% the risk of incapacity to work and in $12 \%$ the risk of hospitalization 29 .

The strength of our study is the random analysis of a community-based population of adults with back pain with a household interview survey adjusted for several potential confounding factors. Population-based studies analyzing HRQoL and back pain are scarce in Brazil 19.

Some methodological limitations should be mentioned, such as the data on the presence of diseases, provided by self-reports of medical diagnosis; however, we used a checklist to increase the accuracy of reporting individual health conditions. The percentage of losses due to refusals or other reasons was not negligible in our study and it tended to differ among variables such as sex, age and socioeconomic level, which can generate selection bias. Non-response weights were then introduced in addition to the sample weights to compensate differential losses according to income, age and sex, and post-stratification weights correcting residual differences in losses by age and sex.

The comparison of scores among studies was difficult regarding the differences in case definition and selection, comorbidities, age, and data presentation. Differences among population studies may occur considering the socioeconomic profile; however, we adjusted it for years of education, which is 
a proxy of economic situation. The cross-sectional design of our study implies that the result must be carefully interpreted and no causal inferences can be made.

Our results showed the major impact of back pain on health-related quality of life. Limitations due to back pain, number of pain locations, pain frequency, and pain intensity have a detrimental impact on the health-related quality of life of people. Moreover, we showed the importance of specific characteristics when assessing back pain impacts on HRQoL.

Studies on general population are much less frequent. Most of the publications are studies with specific groups, mostly about people affected by back pain, assisted by health services such as primary health care, polyclinics, emergency services and hospitals; in "healthy" people, they are focused on occupational groups. Thus, our study sought to fill the existing gaps, and these information could contribute to better understand this reality and seek ways to give better care to patients and prevent these high prevalent diseases.

\section{Contributors}

A. M. Iguti wrote the paper, reviewed the literature, and contributed to the data analysis and discussion. M. G. Lima contributed to the statistical analysis and data discussion, and review the paper. M. B. A. Barros contributed to the study design, data collection, and statistical analysis, and reviewed the paper.

\section{Additional informations}

ORCID: Aparecida Mari Iguti (0000-0002-13097433); Margareth Guimarães Lima (0000-00016996-0745); Marilisa Berti Azevedo Barros (00000003-3974-195X).

\section{Acknowledgments}

The authors are grateful to the São Paulo State Research Foundation (FAPESP) for financing the survey (ISA-Camp 2014/15) and to Brazilian National Research Council (CNPq) for the productivity scholarship provided to M. B. A. Barros. The authors thank to Space of Writing - Pro-Rectory of Research, University of Campinas (Unicamp) for the language services provided.

\section{References}

1. Williams JS, Ng N, Peltzer K, Yawson A, Yawson A, Biritwum R, Maximova T, et al. Risk factors and disability associated with low back pain in older adults in low- and middleincome countries. Results from the WHO Study on Global AGEing and Adult Health (SAGE). PLoS One 2015; 10:e0127880.

2. Larson M. Prevalence of neck and back pain among 45-69 year olds in a general population and the relation to psychosocial and socioeconomic factors. Linköping: Linköping University; 2006.

3. Hoy D, Bain C, Williams G, March L, Brooks P, Blyth F, et al. A systematic review of the global prevalence of low back pain. Arthritis Rheum 2012; 64:2028-37.

4. Deyo RA, Dworkin SF, Amtmann D, Andersson G, Borenstein D, Carragee E, et al. Report of the NIH task force on research standards for chronic low back pain. J Pain 2014; 15:56985.

5. Nilsson E, Kristenson M. Psychological factors related to physical, social, and mental dimensions of the SF-36: a population-based study of middle-aged women and men. Patient Relat Outcome Meas 2010; 1:153-62.

6. Suka M, Yoshida K. Low back pain deprives the Japanese adult population of their quality of life: a questionnaire survey at five healthcare facilities in Japan. Environ Health Prev Med 2008; 13:109-15.

7. Rodríguez-Romero B, Pita-Fernández S, Pértega-Díaz S. Impact of musculoskeletal pain on health-related quality of life among fishing sector workers. Clin Rheumatol 2015; 34:1131-9.

8. Rezai M, Côté P, Cassidy JD, Carroll L. The association between prevalent neck pain and health-related quality of life: a cross-sectional analysis. Eur Spine J 2009; 18:371-81. 
9. Pedisic Z, Pranic S, Jurakic D. Relationship of back and neck pain with quality of life in the Croatian general population. J Manipulative Physiol Ther 2013; 36:267-75.

10. Dominick C, Blyth F, Nicholas M. Patterns of chronic pain in the New Zealand population. N Z Med J 2011; 124:63-76.

11. Nolet PS, Kristman VL, Côté P, Carroll LJ, Cassidy JD, et al. Is low back pain associated with worse health-related quality of life 6 months later? Eur Spine J 2015; 24:458-66.

12. Salaffi F, De Angelis R, Stancati A, Grassi W. Health-related quality of life in multiple musculoskeletal conditions: a cross-sectional population based epidemiological study. II. The MAPPING study. Clin Exp Rheumatol 2005; 23:829-39.

13. Picavet HS, Hoeymans N. Health related quality of life in multiple musculoskeletal diseases: SF-36 and EQ-5D in the DMC3 study. Ann Rheum Dis 2004; 63:723-9.

14. Muraki S, Akune T, Oka H, et al. Health-related quality of life in subjects with low back pain and knee pain in a population-based cohort study of Japanese men: the Research on Osteoarthritis Against Disability study. Spine 2011; 36:1312-9.

15. Tyack Z, Kuys S, Cornwell P, Frakes K-A, McPhail S. Health-related quality of life of people with multimorbidity at a communitybased, interprofessional student-assisted clinic: Implications for assessment and intervention. Chronic Illn 2018; 14:169-81.

16. Alves MCGP, Escuder MML, Goldbaum M Barros MBA, Fisberg RM, Cesar CLG. Sampling plan in health surveys, city of São Paulo, Brazil, 2015. Rev Saúde Pública 2018; 52:81.

17. Laguardia J, Campos MR, Travassos CM, Vasconcellos MM, Najar AL, Anjos LA. Psychometric evaluation of the SF-36 (v.2) questionnaire in a probability sample of Brazilian households: results of the survey Pesquisa Dimensões Sociais das Desigualdades (PDSD), Brazil, 2008. Health Qual Life Outcomes 2011; 9:61.

18. Lima MG, Barros MB, César CL, Goldbaum M, Carandina L, Ciconelli RM. Health related quality of life among the elderly: a populationbased study using SF-36 survey. Cad Saúde Pública 2009; 25:2159-67.

19. Lima MG, Barros MBA, César CLG, Goldbaum M, Carandina L, Ciconelli RM. Impact of chronic disease on quality of life among the elderly in the state of Sao Paulo, Brazil: a population-based study. Rev Panam Salud Pública 2009; 25:314-21.
20. Campolina AG, Lopez RVM, Nardi EP, Ferraz MB. Quality of life in a sample of Brazilian adults using the generic SF-12 questionnaire. Rev Assoc Méd Bras 2018; 64:234-42.

21. Turunen J. Pain and pain management in Finnish general population [Doctoral Dissertation]. Kuopio: Kuopio University; 2007.

22. Leboeuf-Yde C, Fejer R, Nielsen J, Kyvik KO, Hartvigsen J. Pain in the three spinal regions: the same disorder? Data from a populationbased sample of 34,902 Danish adults. Chiropr Man Therap 2012; 20:11.

23. Husky MM, Farin FF, Compagnone P, Fermanian C, Kovess-Masfety V. Chronic back pain and its association with quality of life in a large French population survey. Health Qual Life Outcomes 2018; 16:195.

24. Ogunlana MO, Odunaiya NA, Dairo MD, Ihekuna O. Predictors of health-related quality of life in patients with non-specific low back pain. African Journal of Physiotherapy and Rehabilitation Sciences 2012; 4:15-23.

25. Nolet PS, Côté P, Kristman VL, Rezai M, Carroll LJ, Cassidy JD. Is neck pain associated with worse health-related quality of life 6 months later? A population-based cohort study. Spine J 2015; 15:675-84.

26. Nordstoga AL, Nilsen TIL, Vasseljen O, Unsgaard-Tøndel M, Mork PJ. The influence of multisite pain and psychological comorbidity on prognosis of chronic low back pain: longitudinal data from the Norwegian HUNT Study. BMJ Open 2017; 7:e015312.

27. Tavafian SS, Eftekhar H, Mohammad K, Jamshidi A. Quality of life in women with different intensity of low back pain. Iran J Public Health 2005; 34:36-9.

28. Deng N, Guyer R, Ware Jr. JE. Energym fatigue, or both? A bifactor modeling approach to the conceptualization and measurement of vitality. Qual Life Res 2015; 24:81-93.

29. Ware JE, Kosinski M, Bjorner JB, Gandek B, Turner-Bowker DM, Ware JE. User's manual for the SF36v2 health survey. 2nd Ed. Lincoln: Quality Metric Incorporated; 2007. 


\section{Resumo}

O estudo buscou avaliar o impacto da dor lombar na qualidade de vida relacionada à saúde. $O$ estudo populacional transversal analisou uma amostra de 1.192 adultos. As variáveis dependentes eram as escalas SF-36, e a principal variável independente era dor lombar, caracterizada de acordo com localização, número de regiões com dor lombar, intensidade, frequência e limitações. Foram utilizados modelos de regressão linear simples e multivariada para estimar os coeficientes beta, brutos e ajustados por sexo, idade, escolaridade, comorbidades e doenças crônicas não transmissíveis. A prevalência de dor lombar era $35,4 \%$. Em relação à qualidade de vida relacionada à saúde, a comparação de pessoas com e sem dor lombar mostrou associações fracas para o componente físico $(\beta=-3,6)$. Entretanto, foram encontradas associações fortes para o componente físico $(\beta=-12,4)$ quando havia dor concomitante nas regiões cervical, dorsal e lombar, além de associações com as escalas de saúde mental. A dor diária mostrou associação com os componentes físico $(\beta=-6,8) e$ mental $(\beta=-2,7)$. Foi identificado um impacto importante sobre o escore do domínio físico para dor intensa/muito intensa $(\beta=-7,9)$ e dor com limitação grave $(\beta=-11,5)$. Os impactos sobre qualidade de vida relacionada à saúde eram fortes quando a dor vinha acompanhada de: (1) múltiplos sítios, (2) dor no escore do dominio mental, (3) queixas diárias, (4) dor muito intensa e (5) limitações graves. Os resultados revelaram a importância de analisar fatores específicos relacionados à dor lombar.

Dor nas Costas; Qualidade de Vida; Inquéritos; Epidemiologia

\section{Resumen}

El objetivo de este estudio fue evaluar el impacto del dolor de espalda sobre la calidad de vida, relacionada con la salud. Se trata de un estudio poblacional transversal. Este estudio analizó a 1.192 adultos. Las variables dependientes fueron las escalas SF-36 y las principales variables independientes fueron: dolor de espalda caracterizado por localización, región con número de afectados por dolor de espalda, intensidad, frecuencia y $l i$ mitaciones. Se realizaron modelos de regresión simple y múltiple para estimar los coeficientes beta brutos y ajustados (por género, edad, escolarización y condiciones de comorbilidad), ajustados por género, edad, condiciones de comorbilidad y número de enfermedades crónicas. La prevalencia de dolor de espalda fue 35,4\%. En el caso de calidad de vida, relacionada con la salud, comparando personal con/sin dolor de espalda, hallamos asociaciones débiles relacionadas con el componente físico $(\beta=-3,6)$. No obstante, se encontraron asociaciones fuertes para el componente físico $(\beta=-12,4)$, cuando existía dolor concomitante en las zonas cervicales, dorsales y lumbares y también asociaciones con escalas mentales de salud. El dolor diario estuvo asociado con componentes físicos $(\beta=-6,8)$ y mentales $(\beta=-2,7)$. Se encontró un importante impacto en componente físico por dolor intenso/muy intenso $(\beta=-7,9)$ y dolor con una severa limitación $(\beta=-11,5)$. Los impactos sobre la calidad de vida relacionada con la salud fueron fuertes cuando el dolor de espalda estuvo seguido por (1) múltiples dolores en lugares de la espalda, (2) con dolor en componente mental, (3) quejas diarias, (4) dolor muy intenso y (5) limitaciones severas; estos resultados han revelado la importancia de medir factores específicos relacionados con el dolor de espalda.

Dolor de Espalda; Calidad de Vida; Encuestas; Epidemiología
Submitted on 21/Oct/2019

Final version resubmitted on 18/Jun/2020

Approved on 29/Jun/2020 